\title{
On the cohomology and inner products of the Berkovits superparticle and superstring
}

\author{
Michael Chesterman* \\ The Physics Department, Karlstad University, S-651 88 Karlstad, Sweden.
}

(Dated: April 2004)

\begin{abstract}
We describe the complete cohomology of the Berkovits BRST operator for the superparticle. It is non-zero at eight ghost numbers, splitting into two quartets, the members of each quartet being completely isomorphic. Based only on considerations of the isomorphisms of the cohomology, and using only the standard inner product, we derive the inner product appropriate for string amplitudes. It is in agreement with Berkovits' conjectured prescription, which is one element of an equivalence class. We discuss the Chern-Simons style action for $\mathrm{D}=10$ super Yang-Mills, which is now manifestly superspace covariant.
\end{abstract}

Keywords: BRST quantization, covariant superstring, superspace.

*Electronic address: Michael.Chesterman@kau.se 


\section{INTRODUCTION}

The old problem of the covariant quantization of the superstring has seen some real progress in recent years with Berkovits' approach using pure spinors [1, 2, 3, 4, 5]. See ref. 6] for a review. The interest in a $D=10$ super-Poincaré covariant quantization is largely due to the hope that it can fill in gaps in the computational power of the quantized RNS and light-cone gauge Green-Schwarz strings. These gaps are most notably amplitudes with more than four external fermions, covariant quantization in Ramond-Ramond backgrounds, and higher loop amplitudes.

An unsolved problem in Berkovits' approach is to understand the origin of his conjectured measure, which is used for on-shell calculations such as string amplitudes, and off-shell to construct a cubic string field theory type action for super Yang-Mills and potentially a full open string field theory action [6]. For simplicity, we study the superparticle instead of the superstring wherever possible. The measure is defined for the superparticle indirectly, by the prescription that

$$
\left\langle\left(\lambda \Gamma^{a} \theta\right)\left(\lambda \Gamma^{b} \theta\right)\left(\lambda \Gamma^{c} \theta\right)\left(\theta \Gamma_{a b c} \theta\right)\right\rangle=1,
$$

and roughly speaking expectations of all other covariant combinations of $\theta^{\alpha}$ and $\lambda^{\alpha}$ are zero, where $\theta^{\alpha}$ are fermionic superspace co-ordinates, $\lambda^{\alpha}$ the bosonic pure spinor ghosts, and $\Gamma^{a}$ the gamma matrices.

As well as understanding where the above prescription comes from, it is natural to ask questions, such as whether it is unique. What explicit form should the measure $\mu(\theta, \lambda)$ take? Can we construct a superspace covariant super Yang-Mills, and string field theory action? Note that the prescription in equation (11) isn't manifestly superspace covariant [3] .

We find that the clues needed to answer these questions come from an analysis of the full superparticle cohomology. So far, the cohomology has been studied at positive ghost number [3, 6, 7], where it is believed to be non-zero at ghost numbers $8,9,10,11$, given a convenient definition of the ghost number operator. There is also however non-zero cohomology at ghost numbers $-8,-9,-10,-11$. In my previous work [8, 9], the cohomology at ghost numbers \pm 9 was used to calculate amplitudes for the free superparticle with the standard inner product. However, the cohomology as a whole was not studied. Our interest in the present paper is mainly in the isomorphisms between the cohomologies, and their relation to inner products. 
There is some overlap between this work and the recent paper of Grassi et al. [10], concerning their alternative superstring approach without using pure spinors. A measure is derived using eqn (II) as a starting point, which essentially corresponds with a result derived in this article. However, our approach is rather different, for example we don't assume the Berkovits prescription a priori, but instead derive it.

The paper is organized as follows. In section [I] we study the superparticle cohomology, its isomorphisms and inner products, we similarly study the open string cohomology in section III. we apply these results to open superstring amplitudes in section IV] and to super Yang-Mills and open string field theory in section $\nabla$.

\section{THE COMPLETE SUPERPARTICLE COHOMOLOGY}

\section{A. The Cohomology}

We begin with the phase space for Berkovits' $D=10, \mathcal{N}=1$ superparticle [3], [6]. It has canonical co-ordinates given by $\left(x^{a}, \theta^{\alpha}, \lambda^{\alpha}\right)$ and their respective conjugate momenta $\left(p_{a}, p_{\alpha}, w_{\alpha}\right)$, where $a=0, \ldots 9, \alpha=1, \ldots, 16, \theta^{\alpha}$ is a Majorana-Weyl anticommuting spinor, $\lambda^{\alpha}$ a Weyl commuting complex spinor, and $\left(x^{a}, \theta^{\alpha}\right)$ are the usual superspace co-ordinates. We quantize in the Schrödinger representation where $\left(p_{a}, p_{\alpha}, w_{\alpha}\right) \equiv$ $\left(-i \partial / \partial x^{a},-i \partial / \partial \theta^{\alpha},-i \partial / \partial \lambda^{\alpha}\right)$.

The Berkovits BRST operator is given by

$$
Q=\lambda^{\alpha} D_{\alpha}, \quad Q^{2}=-i \lambda \Gamma^{a} \lambda \frac{\partial}{\partial x^{a}}
$$

where

$$
D_{\alpha} \equiv \frac{\partial}{\partial \theta^{\alpha}}-i \Gamma_{\alpha \beta}^{a} \theta^{\beta} \frac{\partial}{\partial x^{a}}
$$

is the usual superspace covariant derivative, and $\Gamma_{\alpha \beta}^{a}$ are off-diagonal components of the full $32 \times 32$ gamma matrices in the Weyl representation.

In order that $Q$ be nilpotent, the ghosts obey pure spinor constraints

$$
\lambda^{\alpha} \Gamma_{\alpha \beta}^{a} \lambda^{\beta}=0
$$

which are imposed as first class constraints. Observables are required to be gauge invariant with respect to them. There are essentially two approaches available to quantize the ghosts. 
One is by canonical gauge-fixing using $U(5)$ co-ordinates, in a manner analogous to choosing light-cone gauge for the bosonic particle [8, 9]. The second is a BRST approach, in which a second BRST operator $Q_{g c}=C_{a} \lambda \Gamma^{a} \lambda+\ldots$ is introduced to form a BRST double complex 8, 9], where $C_{a}$ are fermionic ghosts with a separate ghost number grading to $\lambda^{\alpha}$ and $w_{\alpha}$, and the ellipses refer to ghosts-for-ghosts terms. There is no known completion of these terms. However, for many applications, we don't need to know them. For the sake of covariance, and not having to constrain $\lambda^{\alpha}$, we use the latter approach.

It was seen in [8, 9] that all conceivable ghosts-for-ghosts terms in $Q_{g c}$ automatically annihilate wave functions at the two 'physical' ghost numbers, which is a typical feature of the Schrödinger representation. Thus, we need only consider the first term of $Q_{g c}$. Generic wave functions take the form $F_{\alpha \beta \ldots}(x, \theta) \lambda^{\alpha} \lambda^{\beta} \ldots \phi_{C=0, U=0}$ and $G^{\alpha \beta \ldots}(x, \theta) w_{\alpha} w_{\beta} \ldots \delta^{(16)}(\lambda) \phi_{B=0, V=0}$, where $C$ and $U$ refer to all the fermionic and bosonic ghosts respectively, $B$ and $V$ to their ghost momenta, and the ghost wave functions are defined as $\phi_{C=0, U=0} \equiv \delta(C) \delta(U)$ and $\phi_{B=0, V=0} \equiv 1$.

We define the ghost number operator

$$
G=\frac{i}{2}\left(\lambda^{\alpha} w_{\alpha}+w_{\alpha} \lambda^{\alpha}\right) \quad G^{\dagger}=-\bar{G},
$$

where $\bar{G}=G(\bar{\lambda}, \bar{w})$, and where $\bar{\lambda}^{\alpha}$ and $\bar{w}_{\alpha}$ correspond to the complex conjugates of $\lambda^{\alpha}$ and $w_{\alpha}$ respectively. Differently to [8, 9], we ignore the extra ghost terms, preempting that we will factor out the $C$ and $U$ ghost wave function from physical states. As shown later in this section, the generalized antihermicity condition obeyed by $G$ ensures that the state cohomology forms doublets at ghost number $\pm g$.

The upshot of the BRST approach, after factorizing out the $C$ and $U$ ghost wave functions for simplicity, is that there are two different definitions of physical states, depending on the ghost number. For $g \geq 8$, a physical state $\psi_{g}$ at ghost number $g$ is defined by

$$
Q \psi_{g} \approx 0, \quad \delta \psi_{g} \approx Q \psi_{g-1}
$$

where

$$
\psi_{1} \approx \psi_{2} \Rightarrow \psi_{1}=\psi_{2}+\lambda \Gamma^{a} \lambda \phi_{a}
$$

for some wave function $\phi_{a}$. Whereas for $g \leq 8$,

$$
\begin{gathered}
\lambda \Gamma^{a} \lambda \psi_{g}=0, \quad Q \psi_{g}=0, \\
\delta \psi_{g}=Q \psi_{g-1}, \quad \lambda \Gamma^{a} \lambda \psi_{g-1}=0 .
\end{gathered}
$$


Note that there are no wave functions with $-8<g<8$, as is standard for bosonic ghosts. For negative ghost number, the approach with the ghost constraints is essentially the same as Dirac's old covariant approach.

Some typical wave functions are

$$
\psi_{-10}=\omega_{\alpha} \omega_{\beta} A^{\alpha \beta}(x, \theta) \delta^{(16)}(\lambda), \quad \psi_{11}=\lambda^{\alpha} \lambda^{\beta} \lambda^{\gamma} \tilde{C}_{\alpha \beta \gamma}(x, \theta) .
$$

The equations of motion and gauge transformation of $A^{\alpha \beta}(x, \theta)$ coming from (8) and (9) are

$$
\begin{gathered}
\Gamma_{\alpha \beta}^{a} A^{\alpha \beta}=0, \quad D_{\alpha} A^{\alpha \beta}=0, \\
\delta A^{\alpha \beta}=D_{\gamma} \phi^{\alpha \beta \gamma}, \quad \Gamma_{\alpha \beta}^{a} \phi^{\alpha \beta \gamma}=0,
\end{gathered}
$$

where $\phi^{\alpha \beta \gamma}$ is an arbitrary superfield, and all the superfields are symmetric in their spinor indices. It is more elegant to keep the ghosts $\lambda^{\alpha}$ when describing the equations of motion and gauge transformations of superfields at positive ghost number

$$
\lambda^{\alpha} \lambda^{\beta} \lambda^{\gamma} \lambda^{\delta} D_{\alpha} \tilde{C}_{\beta \gamma \delta} \approx 0, \quad \lambda^{\alpha} \lambda^{\beta} \lambda^{\gamma} \delta \tilde{C}_{\alpha \beta \gamma} \approx \lambda^{\alpha} \lambda^{\beta} \lambda^{\gamma} D_{\alpha} \phi_{\beta \gamma}
$$

where $\phi_{\alpha \beta}$ is an arbitrary superfield.

The complete state cohomology $H^{*}(Q)$ is summarised below in table @

TABLE I: The Superparticle Cohomology

\begin{tabular}{|c|c|c|c|}
\hline Ghost number & Wave function & Physical content & $\theta$ levels \\
\hline-11 & $C^{\alpha \beta \gamma}(x, \theta)$ & $c(x)$ & 11 \\
\hline-10 & $A^{\alpha \beta}(x, \theta)$ & $a_{a}(x), \chi^{\alpha}(x)$ & 12,13 \\
\hline-9 & $\tilde{A}^{\alpha}(x, \theta)$ & $\tilde{a}^{a}(x), \tilde{\chi}_{\alpha}(x)$ & 15,14 \\
\hline-8 & $\tilde{C}(x, \theta)$ & $\tilde{c}(x)$ & 16 \\
\hline 8 & $C(x, \theta)$ & $c(x)$ & 0 \\
\hline 9 & $A_{\alpha}(x, \theta)$ & $a_{a}(x), \chi^{\alpha}(x)$ & 1,2 \\
\hline 10 & $\tilde{A}_{\alpha \beta}(x, \theta)$ & $\tilde{a}^{a}(x), \tilde{\chi}_{\alpha}(x)$ & 4,3 \\
\hline 11 & $\tilde{C}_{\alpha \beta \gamma}(x, \theta)$ & $\tilde{c}(x)$ & 5 \\
\hline
\end{tabular}


where

$$
\begin{aligned}
& \partial_{a} c(x)=0, \quad \delta \tilde{c}(x)=\partial_{a} b^{a}(x), \\
& \partial^{a}\left(\partial_{a} a_{b}-\partial_{b} a_{a}\right)=0, \quad \delta \tilde{a}^{a}=\partial^{b}\left(\partial_{a} s_{b}(x)-\partial_{b} s_{a}(x)\right), \\
& \partial_{a} \tilde{a}^{a}=0, \quad \delta a_{a}(x)=\partial_{a} \phi(x), \\
& \Gamma_{\alpha \beta}^{a} \partial_{a} \chi^{\beta}=0, \quad \delta \tilde{\chi}_{\alpha}=\Gamma_{\alpha \beta}^{a} \partial_{a} \phi^{\beta}(x),
\end{aligned}
$$

and where $b^{a}, s_{a}, \phi, \phi^{\beta}$ are arbitrary functions of $x$. The cohomology at all other ghost numbers is null. We show only the superfield part of the wave functions, since the ghost part can be easily deduced from eqn (10). The fourth column for $\theta$ level tells us at what power of $\theta$ the physical content lies. The physical content of the cohomology is given by two copies of the on-shell fields of super-Maxwell theory in the Batalin-Vilkovisky formalism. These are the photon, photino and ghost fields $a_{a}(x), \chi^{\alpha}(x), c(x)$ and their respective antifields $\tilde{a}^{a}(x), \tilde{\chi}_{\alpha}(x)$ and $\tilde{c}(x)$.

The positive ghost number cohomology was first deduced in [3, 6], including a proof that $H^{g}(Q)$ is null for $g>11$. Also, a direct computation of the representation content of this cohomology was made [7] using the computer program LiE [11], which agrees with Berkovits' results. Below is given a convenient representative of each cohomology class at positive ghost number

$$
\begin{aligned}
C(x, \theta) & =c \\
\lambda^{\alpha} A_{\alpha}(x, \theta) & \sim a_{a}(x) \lambda \Gamma^{a} \theta+\left(\chi(x) \Gamma_{a b c} \lambda\right)\left(\theta \Gamma^{a b c} \theta\right)+\ldots, \\
\lambda^{\alpha} \lambda^{\beta} \tilde{A}_{\alpha \beta}(x, \theta) & \sim \lambda \Gamma^{a b c d e} \lambda\left[\left(\theta \Gamma_{a b c} \theta\right)\left(\theta \Gamma_{d e}\right)^{\alpha} \tilde{\chi}_{\alpha}(x)+\left(\theta \Gamma_{a b c} \theta\right)\left(\theta \Gamma_{d e f} \theta\right) \tilde{a}^{f}(x)+\ldots\right] \\
\lambda^{\alpha} \lambda^{\beta} \lambda^{\gamma} \tilde{C}_{\alpha \beta \gamma}(x, \theta) & \sim \tilde{c}\left(\lambda \Gamma^{a} \theta\right)\left(\lambda \Gamma^{b} \theta\right)\left(\lambda \Gamma^{c} \theta\right)\left(\theta \Gamma_{a b c} \theta\right),
\end{aligned}
$$

where $c$ and $\tilde{c}$ are constants, and $\psi \sim \psi^{\prime} \Rightarrow \psi=\psi^{\prime}+Q \phi$ for some wave function $\phi$. We argue later in section $\llbracket B$ that we can always choose a Wess-Zumino type gauge such that the superfields $A_{\alpha}$ and $\tilde{A}_{\alpha \beta}$ terminate at $\theta$-level five.

The negative ghost number cohomology follows from the above since the cohomology $H^{-g}(Q)$ is isomorphic to $H^{g}(Q)$ for each $g$, as a result of the usual non-degenerate, welldefined inner product. For a discussion, see [8, 9, 12]. Roughly speaking, a basis of BRSTclosed but not exact states $\left\{\psi_{g}^{A}\right\}$ can be chosen, such that

$$
\left\langle\bar{\psi}_{g}^{B} \mid \psi_{g^{\prime}}^{A}\right\rangle=\delta^{A B} \delta_{g+g^{\prime}}
$$


where $\bar{\psi}_{g}=\psi_{g}(\bar{\lambda}, x, \theta)$ and $\left\{\psi_{g}^{A}\right\}$ are orthogonal to all other states. So $\psi_{g}^{A}$ maps to $\bar{\psi}_{-g}^{A}$ under the isomorphism. As in [8, 9], we place barred states on the left in the inner product. One can see that states at ghost number $g$ couple to states at ghost number $-g$ for finite inner product, since by considering $\left\langle\bar{\psi}_{g}|G| \psi_{g^{\prime}}\right\rangle$ with eqn (15), we find

$$
\left(g+g^{\prime}\right)\left\langle\bar{\psi}_{g} \mid \psi_{g^{\prime}}\right\rangle=0
$$

The general prescription to calculate $H^{-g}(Q)$ given $H^{g}(Q)$ is that a field at $\theta$-level $l$ in $\psi_{g}$ becomes its anti-field at $\theta$-level $16-l$ in $\psi_{-g}$. Note that under the inner product, $(\theta)^{l}$ couples to $(\theta)^{16-l}$ due to the integration $\int d^{16} \theta$. So $\theta^{\alpha}$ is replaced by $\partial / \partial \theta^{\alpha}$ and $\lambda^{\alpha}$ by $\partial / \partial \lambda^{\alpha}$, with a $\delta^{16}(\theta) \delta^{16}(\lambda)$ placed at the end, where $\delta^{(16)} \theta \equiv \prod_{\alpha} \theta^{\alpha}$. As an example

$$
\psi_{-11} \sim c\left(\omega \Gamma^{a} \tilde{\theta}\right)\left(\omega \Gamma^{b} \tilde{\theta}\right)\left(\omega \Gamma^{c} \tilde{\theta}\right)\left(\tilde{\theta} \Gamma_{a b c} \tilde{\theta}\right) \delta^{(16)}(\lambda)
$$

where $c \in \mathbb{C}$ is constant and

$$
\tilde{\theta}_{\alpha} \tilde{\theta}_{\beta} \ldots \equiv \frac{\partial}{\partial \theta^{\alpha}} \frac{\partial}{\partial \theta^{\beta}} \ldots \delta^{(16)}(\theta)
$$

\section{B. Isomorphisms and inner products}

We have discussed the isomorphisms between $H^{g}(Q)$ and $H^{-g}(Q)$. However, there are further isomorphisms, which are perhaps unexpected from a BRST point of view, since they are specific to this system. From table I we see that there are only two independent cohomologies $H^{8}(Q)$ and $H^{9}(Q)$. All others are isomorphic to one or the other. Specifically

$$
\begin{array}{r}
H^{8}(Q) \cong H^{-8}(Q) \cong H^{11}(Q) \cong H^{-11}(Q) \cong \mathbb{C}, \\
H^{9}(Q) \cong H^{-9}(Q) \cong H^{10}(Q) \cong H^{-10}(Q) \cong \mathbf{8}+\mathbf{8},
\end{array}
$$

where $\mathbf{8}+\mathbf{8}$ refers to the on-shell degrees of freedom of super Yang-Mills.

Since the isomorphism $H^{g}(Q) \cong H^{-g}(Q)$ is associated with the standard inner product, we might expect to find other inner products associated with other isomorphisms. This is indeed the case. However, rather than looking for such inner products, we look for explicit one-to-one maps between cohomologies and use the standard inner product. For example, to find the inner product relating $H^{9}(Q)$ to $H^{10}(Q)$, we first look for a map $f: H^{9}(Q) \rightarrow H^{-10}(Q)$, then $H^{-10}(Q)$ couples to $H^{10}(Q)$ under the standard inner product. 
In order to find such a mapping, first note that the BRST invariant wave function $\lambda^{\alpha} A_{\alpha}$ is also a BRST invariant operator, since $Q \lambda^{\alpha} A_{\alpha} \equiv\left[Q, \lambda^{\alpha} A_{\alpha}\right]$. The same is true for all wave functions with $g \geq 8$. One can therefore think of $\psi_{9}$ as the BRST invariant operator $\lambda^{\alpha} A_{\alpha}$ acting on the BRST invariant vacuum state $\psi_{8}(1) \equiv 1$

$$
\psi_{9}\left(a_{a}, \chi^{\alpha}\right)=\lambda^{\alpha} A_{\alpha}\left(a_{a}, \chi^{\alpha}\right) \psi_{8}(1)
$$

where we write BRST-closed states $\psi_{g}$ as functions of their physical content, as described in eqns (18) to (21). Other BRST-closed states for $g \geq 8$ are built in a similar manner.

We can now construct different BRST-invariant states by replacing the vacuum state. States in any of the cohomologies isomorphic to $H^{8}(Q)$ in eqn (26) are candidates. We choose $\psi_{-11}(1)$, where $\psi_{-11}$ is given in equation (24). The claim is then that

$$
\begin{aligned}
& \psi_{-11}(c) \sim C(c) \psi_{-11}(1), \psi_{-10}\left(a_{a}, \chi^{\alpha}\right) \sim \lambda^{\alpha} A_{\alpha}\left(a_{a}, \chi^{\alpha}\right) \psi_{-11}(1), \\
& \psi_{-9}\left(\tilde{a}^{a}, \tilde{\chi}_{\alpha}\right) \sim \lambda^{\alpha} \lambda^{\beta} \tilde{A}_{\alpha \beta}\left(\tilde{a}^{a}, \tilde{\chi}_{\alpha}\right) \psi_{-11}(1), \quad \psi_{-8}(\tilde{c}) \sim \lambda^{\alpha} \lambda^{\beta} \lambda^{\gamma} \tilde{C}_{\alpha \beta \gamma}(\tilde{c}) \psi_{-11}(1),
\end{aligned}
$$

at least up to a rescaling of the physical fields, which can be taken care of by calculating suitable factors in the superfield expansions of eqns (18) to (21). This is reminiscent of the mapping $c: H^{-1 / 2}(Q) \rightarrow H^{1 / 2}(Q)$ for the bosonic particle, where $c$ is the worldline reparameterization ghost.

A direct calculation of the above is feasible but time-consuming, except in the case of $\psi_{-11}(c)$. However, we can see why it must be true indirectly. Consider the expression for $\psi_{-10}$ in (29). From the point of view of the physical content of $\psi_{-10}$, the scalar $c$ in $\psi_{-11}(c)$ is at $\theta$-level 11, and the vector and spinor $a_{a}$ and $\chi^{\alpha}$ are at levels one and two respectively in $\lambda^{\alpha} A_{\alpha}$. This leads to $a_{a}$ and $\chi^{\alpha}$ being at levels 12 and 13 in $\psi_{-10}$, which is consistent with table I. Given that $\lambda^{\alpha} A_{\alpha}$ and $\psi_{-11}$ are BRST-closed but not BRST-exact, the same must be the case for $\psi_{-10}$, and hence the physical fields $a_{a}$ and $\chi^{\alpha}$ in $\lambda^{\alpha} A_{\alpha}$ must map to the $a_{a}$ and $\chi^{\alpha}$ in $\psi_{-10}$ since there are no other candidate physical fields. Similar arguments apply at the other ghost numbers.

We now have a non-degenerate inner product between the cohomologies $H^{g}(Q)$ and $H^{13-g}(Q)$ for $8 \leq g \leq 11$, which will be useful later for string amplitude and string field theory calculations. Defining the vacuum states

$$
|0\rangle \equiv 1, \quad|\Omega\rangle \sim \psi_{-11}(1),
$$


the inner product between states/operators $\psi$ and $\phi$ is written

$$
\langle\bar{\phi} \bar{\Omega} \mid \psi\rangle=\left\langle\bar{\Omega}\left|\bar{\phi}^{\dagger} \psi\right| 0\right\rangle
$$

In the case where the $(x, \theta)$ part of the wave function $\phi$ is real, $\bar{\phi}^{\dagger}=\phi$. The vacuum states are supersymmetric in the sense that

$$
Q_{\alpha}|0\rangle=0, \quad Q_{\alpha}|\Omega\rangle \sim 0
$$

where $Q_{\alpha}$ is the supersymmetry generator

$$
Q_{\alpha} \equiv \frac{\partial}{\partial \theta^{\alpha}}+i \Gamma_{\alpha \beta}^{a} \theta^{\beta} \frac{\partial}{\partial x^{a}}, \quad\left\{Q_{\alpha}, Q\right\}=0
$$

We know that $Q_{\alpha} \mid \Omega>$ is BRST-exact because it is BRST-closed, and using the representative $\psi_{-11}(1)$ in eqn (24) for $|\Omega\rangle$, must consist of only one term at $\theta$-level 10, which does not correspond to the only non-exact but closed state in (24).

As an example, the inner product between the BRST closed states $\psi_{9}$ and $\psi_{10}$ is

$$
\left\langle\bar{\Omega}\left|\lambda^{\alpha} \lambda^{\beta} \tilde{A}_{\alpha \beta}^{\dagger} \lambda^{\gamma} A_{\gamma}\right| 0\right\rangle=\left\langle\psi_{-9}\left(\tilde{a}^{a}, \tilde{\chi}_{\alpha}\right) \mid \psi_{9}\left(a_{a}, \chi^{\alpha}\right)\right\rangle \propto \int d^{10} x\left(\left(\tilde{a}^{a}\right)^{*} a_{a}+\left(\tilde{\chi}_{\alpha}\right)^{*} \chi^{\alpha}\right)
$$

where the last equality is explained in $[8,9]$. Note that this expression is invariant under $\delta \Omega=$ $Q \psi_{-12}$, as well as possessing the usual BRST symmetries and supersymmetry with respect to $\lambda^{\alpha} \lambda^{\beta} \tilde{A}_{\alpha \beta}$ and $\lambda^{\gamma} A_{\gamma}$. In the case where we specifically take the representative for $|\Omega\rangle$ in eqn (24), $\langle\bar{\Omega}|$ couples only to the BRST-closed term in eqn (21) under the standard inner product, which agrees precisely with Berkovits' prescription in (11). In fact this prescription is one element of an equivalence class. Replacing $|\Omega\rangle$ with $|\Omega\rangle+Q|\phi\rangle$ for general state $|\phi\rangle$, we obtain the most general prescription.

An interesting observation which follows from the mapping in (29) and (30), is that given the explicit choice for $\psi_{-11}(1)$ in eqn (24) which has $11 \theta$ 's, all the wave functions $\psi_{g}, g=-8, \ldots,-11$ terminate at $\tilde{\theta}$-level 5 . This means that through the isomorphism $H^{-g}(Q) \cong H^{g}(Q)$, a cohomology class representative $\psi_{g}, g=8, \ldots, 11$ can always be chosen which terminates at $\theta$-level 5 . So it is quite feasible to calculate the few remaining terms of $A_{\alpha}$ and $\tilde{A}_{\alpha \beta}$ in eqns (19) and (20).

It is in principle possible to calculate inner products associated with the remaining isomorphisms. In particular, following an approach analogous to above, we may construct an inner product which couples $H^{g}(Q)$ to $H^{-19-g}(Q)$ for $g=-8, \ldots,-11$. It is a little more 
complicated however, since the BRST-closed wave function $\omega_{\alpha} \tilde{A}^{\alpha}\left(p_{a}, p_{\alpha}\right)$ in the momentum picture isn't also a BRST-closed operator. Any analogous inner product between $H^{9}(Q)$ and $H^{-10}(Q)$ would not be Lorentz covariant because we cannot form a Lorentz scalar from two $\chi^{\alpha}$ 's.

\section{ON THE SUPERSTRING COHOMOLOGY}

The above ideas extend fairly easily to the case of the superstring, where the superparticle degrees of freedom form its zero modes. We conjecture that the complete open string cohomology takes the same form as the superparticle cohomology, in the sense that cohomology at the different ghost numbers play exactly the same roles. For example $H^{8}(Q)$ will contain all on-shell space-time Batalin-Vilkovisky ghosts, $H^{11}(Q)$ all the anti-ghosts etc., where now $Q=\oint d z i \lambda^{\alpha} d_{\alpha}$, with $d_{\alpha}=p_{\alpha}-i\left(\partial X_{a}-\frac{i}{2} \theta \Gamma_{a} \theta\right)\left(\Gamma^{a} \theta\right)_{\alpha}$. This is a natural conjecture to make in view of the strong analogy noted in [3] , between the Berkovits string and topological strings [13], which have precisely this cohomology structure. However, except for the ghost number one vertex operator cohomology $H_{\mathrm{op}}^{1}(Q) \equiv H^{9}(Q)$ which is known to give the correct string spectrum [5, 14], no direct calculations of cohomology at non-zero excitation level have been made. By reversing the logic of the previous subsection, we nevertheless prove that the isomorphisms of eqns (26) and (27) also hold for the superstring.

The generalization of the relation (29) for the state mapping $\psi_{9} \rightarrow \psi_{-10}$ is given by

$$
\psi_{9}=V(0)|0\rangle \quad \psi_{-10} \sim V(0) \Omega(0)|0\rangle
$$

where $V(z) \in H_{\mathrm{op}}^{1}(Q)$ is a ghost number one BRST-closed unintegrated vertex operator, which is $\lambda^{\alpha} A_{\alpha}$ for the open string ground state,

$$
\Omega(z) \sim \frac{1}{(2 \pi)^{16}} \int d^{16} u\left(u \Gamma^{a} \tilde{\theta}\right)\left(u \Gamma^{b} \tilde{\theta}\right)\left(u \Gamma^{c} \tilde{\theta}\right)\left(\tilde{\theta} \Gamma_{a b c} \tilde{\theta}\right) e^{i u_{\alpha} \lambda^{\alpha}}
$$

and $\tilde{\theta}, \lambda$ are as usual all holomorphic functions of $z$. The vacuum $|0\rangle$ is the usual one associated with 1 in the state-operator correspondence. It is the direct product of the wave function $\psi_{8}(1)=1$ for the zero modes, which contributes ghost number 8, with the Fock space vacuum for the non-zero modes, which contributes ghost number 0 . Note also that the ghost number -11 vacuum state $|\Omega\rangle \equiv \Omega(0)|0\rangle$ corresponds to a direct product of the wave function $\psi_{-11}(1)$ in eqn (24) for the zero modes, with the Fock space vacuum for the non-zero modes. 
Since the string cohomologies have infinite size, we must prove that the isomorphisms hold true at each level of excitation $n \geq 0$. A linearly independent basis of BRST-closed but not BRST-exact vertex operators $V(z)$ at excitation level $n$ must map to a basis of states $\psi_{-10}$ with these same properties, since $|\Omega\rangle$ is a BRST-closed but not BRST-exact state at level 0. Thus, $H_{n}^{9}(Q) \subseteq H_{n}^{-10}(Q)$, where $H_{n}^{*}(Q)$ is the subcohomology with excitation level $n$. Using similar arguments for the mapping $\psi_{10} \rightarrow \psi_{-9}$, we find also that $H_{n}^{10}(Q) \subseteq H_{n}^{-9}(Q)$. Now we know that $H_{n}^{9}(Q) \cong H_{n}^{-9}(Q)$ and $H_{n}^{10}(Q) \cong H_{n}^{-10}(Q)$, due to the standard non-degenerate inner product as discussed in section [I] Thus, we conclude

$$
H^{9}(Q) \cong H^{-9}(Q) \cong H^{10}(Q) \cong H^{-10}(Q)
$$

as expected. A similar argument can be used to show that

$$
H^{8}(Q) \cong H^{-8}(Q) \cong H^{11}(Q) \cong H^{-11}(Q)
$$

\section{TREE-LEVEL OPEN SUPERSTRING AMPLITUDES}

For free string calculations, we may use either the standard inner product, or a generalization of the inner product in (32) $\left\langle\bar{\Omega}\left|\bar{V}_{1}^{\dagger}(0) V_{2}(0)\right| 0\right\rangle$, where $|\Omega\rangle \equiv \Omega(0)|0\rangle$ is the ghost number -11 vacuum state, and $V_{i}(z)$ are unintegrated vertex operators.

The expression for tree-level amplitudes is a modification of Berkovits' expression [6], though now just using the ordinary inner product between two different vacuum states

$$
A=\operatorname{tr}\left\langle\bar{\Omega}\left|V_{1}\left(z_{1}\right) V_{2}\left(z_{2}\right) V_{3}\left(z_{3}\right) U_{4} \ldots U_{n}\right| 0\right\rangle
$$

where $U_{i}=\oint d z u_{i}(z)$ are integrated vertex operators, which are related to their unintegrated couterparts as $\left[Q, u_{i}\right]=\partial_{z} V_{i}$. Note that the ghost numbers of the two vacua sum to -3 , which is an important check. Similarly to the superparticle analysis in section IIB given the cohomology class representative for $\Omega(z)$ in eqn (37), the above expression corresponds exactly with Berkovits' prescription in equation (11). It is invariant under $\delta|\Omega\rangle=Q\left|\psi_{-12}\right\rangle$ for arbitrary $\left|\psi_{-12}\right\rangle$, so that Berkovits' prescription is one of a BRST equivalence class.

All symmetries are manifest in the above expression. $A$ is manifestly BRST invariant. It is also manifestly invariant under superspace translations, since in particular as a generalization of (33), the vacuum states obey

$$
\oint d z q_{\alpha}(z)|0\rangle=0, \quad \oint d z q_{\alpha}(z)|\Omega\rangle \sim 0
$$


where $q_{\alpha}=p_{\alpha}+i\left(\partial X_{a}-\frac{i}{6} \theta \Gamma_{a} \partial \theta\right) \Gamma_{\alpha \beta}^{a} \theta^{\beta}$ and $\oint d z q_{\alpha}(z)$ is the supersymmetry generator. Furthermore it is $S L(2, \mathbb{R})$ covariant since

$$
L_{0, \pm 1}|0\rangle=0, \quad L_{0, \pm 1}|\Omega\rangle \sim 0
$$

and the unintegrated vertex operators $V_{i}\left(z_{i}\right)$ have zero conformal weight. The final statement of (42) requires a proof, but it is similar to the result that $P_{a} P^{a} \psi_{9} \sim 0$ for BRST-closed $\psi_{9}$ in the case of the superparticle as shown in [8, 9].

There is a complication that massive states in general have vertex operators with terms which need a BRST extension with respect to the ghost constraint BRST operator $Q_{g c}=$ $C_{a} \lambda \Gamma^{a} \lambda+\ldots$, like $\lambda^{\alpha} w_{\alpha}$. This is a problem, because there is currently no known completion

of the ghost terms. However, the calculation may still be doable using methods in [8, 9$]$. Further investigation is warranted. Finally, it seems reasonable that amplitudes involving closed strings will be a straightforward generalization of this.

\section{A SUPERSPACE ACTION FOR $D=10$ SUPER YANG-MILLS}

We now apply the technique of the previous section to Berkovits' Chern-Simons style action for super Yang-Mills. The result is not as nice as for on-shell calculations like string amplitudes, essentially because while the inner product described in eqn (32) is non-degenerate on-shell, it is highly degenerate off-shell. This is clear since any state with more than $5 \theta$ 's is orthogonal to all other states, taking the representative for $|\Omega\rangle$ in eqn (24).

That being said, the BRST invariant action is

$$
S=\operatorname{tr} \frac{1}{2}\langle\bar{\Omega}|\psi Q \psi| 0\rangle+\operatorname{tr} \frac{g}{3}\langle\bar{\Omega}|\psi \psi \psi| 0\rangle
$$

where

$$
\psi=C(x, \theta)+\lambda^{\alpha} A_{\alpha}(x, \theta)+\lambda^{\alpha} \lambda^{\beta} \tilde{A}_{\alpha \beta}(x, \theta)+\lambda^{\alpha} \lambda^{\beta} \lambda^{\gamma} \tilde{C}_{\alpha \beta \gamma}(x, \theta),
$$

and the Lie algebra indices have been suppressed. The equations of motion are

$$
(Q \psi+g \psi \cdot \psi)|\Omega\rangle=0
$$

which is a much less restrictive equation than the actual equation for super Yang-Mills $Q \psi+g \psi \cdot \psi=0$. Unlike the string amplitude calculation, the above action and equations of motion vary under $\delta|\Omega\rangle=Q\left|\psi_{-12}\right\rangle$. It was argued in [3], using Berkovits' prescription which 
corresponds with choosing the exact representative for $|\Omega\rangle=\psi_{-11}(1)$ given in eqn (24), that after integrating out $\theta^{\alpha}$ and $\lambda^{\alpha}$, the action (43) is the standard BV one except with no auxiliary field terms. Therefore, the equations of motion (45) must imply no conditions on the auxiliary fields, effectively meaning that all the auxiliary fields have become pure gauge. By making a different choice of $|\Omega\rangle$, the action is modified and auxiliary field terms appear. The precise implications of this are yet to be understood. Another way to understand this issue, is that one can write

$$
\delta S=\langle\delta \bar{\psi} \bar{\Omega}|(Q \psi+g \psi \cdot \psi)| 0\rangle=0
$$

for every $\delta \psi$. Consider the case $\delta \psi=\lambda^{\alpha} \delta A_{\alpha}$. The mapping $\delta A_{\alpha} \rightarrow \delta A^{\alpha \beta}$ given by $\delta \psi \rightarrow$ $\delta \psi|\Omega\rangle$ is one-to-one on-shell, but off-shell only a fraction of the functional space spanned by $\delta A^{\alpha \beta}$ is covered by the map, largely because the representation content of $A_{\alpha}$ is much smaller than the representation content of $A^{\alpha \beta}$. Thus, $\langle\delta \bar{\psi} \bar{\Omega}|$ covers only a fraction of the ghost number -10 state space.

Integrating out the ghosts, the action becomes

$$
S=\operatorname{tr} \int d^{10} x d^{16} \theta C^{\alpha \beta \gamma}(1)\left(\frac{1}{2} A_{\alpha} D_{\beta} A_{\gamma}+\frac{g}{3} A_{\alpha} A_{\beta} A_{\gamma}+\text { BV ghost terms }\right),
$$

where

$$
C^{\alpha \beta \gamma}(1) \sim\left(\Gamma^{a} \tilde{\theta}\right)^{\alpha}\left(\Gamma^{b} \tilde{\theta}\right)^{\beta}\left(\Gamma^{c} \tilde{\theta}\right)^{\gamma}\left(\tilde{\theta} \Gamma_{a b c} \tilde{\theta}\right)
$$

is a non-dynamical superfield. The main advantage of this expression over that of Berkovits is that the action is now superspace covariant. Under supersymmetry transformations we allow $C^{\alpha \beta \gamma}$ to transform just as the other superfields $\delta_{\varepsilon} C^{\alpha \beta \gamma}=\left[\varepsilon^{\rho} Q_{\rho}, C^{\alpha \beta \gamma}\right]$, where $\varepsilon^{\rho}$ is a Grassmann parameter. The superspace transformation of $C^{\alpha \beta \gamma}$ cancels auxiliary field terms in the variation of physical fields. For example $\delta_{\varepsilon} a_{a}=\left(i \varepsilon \Gamma_{a} \chi+\right.$ aux. field terms $)$, where these auxiliary field terms vanish on-shell. Recall that the action $S$ is independent of auxiliary fields for suitable choice of $C^{\alpha \beta \gamma}$. While the action is superspace covariant, it has the unusual features of a non-dynamical superfield $C^{\alpha \beta \gamma}$ and missing auxiliary field terms. It will be interesting to see how it can be applied in a string field theory context. In particular, it is desirable to understand better the role of $C^{\alpha \beta \gamma}$.

The full cubic string field theory action is a fairly straightforward generalization of the super Yang-Mills action

$$
S=\operatorname{tr}\left\langle\bar{\Omega}\left|\left(\frac{1}{2} V Q V+\frac{g}{3} V V V\right)\right| 0\right\rangle
$$


where $V$ is the sum of ghost number $0,1,2$ and 3 vertex operators.

\section{CONCLUDING REMARKS}

It is interesting to note that the fact that an inner product exists which is suitable for string amplitudes is a result of the isomorphism structure of the cohomology. In particular, that we can find two suitable vacuum states whose ghost number sums to -3 suggests that somehow the cohomology structure of $Q$ 'knows' about string tree-level amplitudes.

\section{Acknowledgments}

I would like to thank Anders Westerberg for many helpful discussions and for reading the manuscript.

[1] N. Berkovits, JHEP 04, 018 (2000), hep-th/0001035.

[2] N. Berkovits and B. C. Vallilo, JHEP 07, 015 (2000), hep-th/0004171.

[3] N. Berkovits, JHEP 09, 016 (2001), hep-th/0105050.

[4] N. Berkovits and O. Chandia, Phys. Lett. B514, 394 (2001), hep-th/0105149.

[5] N. Berkovits and O. Chandia (2002), hep-th/0204121.

[6] N. Berkovits (2002), hep-th/0209059.

[7] M. Cederwall, B. E. W. Nilsson, and D. Tsimpis, JHEP 02, 009 (2002), hep-th/0110069.

[8] M. Chesterman, JHEP 02, 011 (2004), hep-th/0212261.

[9] M. Chesterman, Ph.D. thesis, Queen Mary, London (2002).

[10] P. A. Grassi, G. Policastro, and P. van Nieuwenhuizen (2004), hep-th/0402122.

[11] A. Cohen, M. van Leeuwen, and B. Lisser, LiE v2.2 (1998), URL http://young.sp2mi.univ-poitiers.fr/〜marc/LiE/

[12] M. Henneaux and C. Teitelboim, Quantization of gauge systems (1992), princeton, USA: Univ. Pr. 520 p.

[13] E. Witten (1992), hep-th/9207094.

[14] N. Berkovits, JHEP 09, 046 (2000), hep-th/0006003. 